\title{
A Comparative Study of Antidiabetic Activity of Hexane-Extract of Lemon Peel (Limon citrus) and Glimepiride in Alloxan-Induced Diabetic Rats
}

\author{
Mohammad Naim ${ }^{1}$, Farhad Mohammad Amjad ${ }^{2}$, Sania Sultana ${ }^{2}$, Sheikh Nazrul Isalm ${ }^{3}$, Muhammad Amjad \\ Hossain $^{1}$, Rehana Begum ${ }^{1}$, Mohammad Abdur Rashid ${ }^{1}$ and Mohammad Shah Amran ${ }^{1}$ \\ ${ }^{1}$ Department of Pharmaceutical Chemistry, Faculty of Pharmacy, University of Dhaka, Dhaka, Bangladesh \\ ${ }^{2}$ Dhaka Medical College, Dhaka, Bangladesh \\ ${ }^{3}$ Institute of Nutrition and Food Sciences, Dhaka University, Dhaka, Bangladesh.
}

\begin{abstract}
Diabetes is classified as a metabolic disorder manifested by elevated level of glucose in the blood. An attempt has been made to compare the antidiabetic activity of hexane extract of lemon peel (Citrus Limon) with that of a marketed product, glimepiride, in alloxan-induced diabetic rats. The study was performed by the measurement of blood glucose level using a glucometer. It was found that the hexane extract of lemon peel showed antidiabetic activity comparable to that of glimepiride. It can thus be inferred that the extract of lemon peel possesses significant antidiabetic activity.
\end{abstract}

Key words: Diabetic, Alloxan, glimepiride, lemon peel, glucometer.

\section{Introduction}

Diabetes is classified as a metabolic disorder. Metabolism refers to the way our bodies use digested food for energy and growth. Most of what we eat is broken down into glucose which is a form of sugar in the blood. It is the main source of fuel for our bodies. Glucose cannot enter our cells without insulin. Lemon contains a class of organic substance called polyphenols that are present in high amount in the lemon peel. These dietary lemon polyphenols were fed to obese experimental animals to study the effects of polyphenols on obesity. It was found from the study that polyphenols significantly suppressed different aspects of obesity such as weight gain, fat accumulation, development of hyperlipidemia, high blood glucose levels and insulin resistance. Citrus peel contains a range of essential oils. These essential oils are found to inhibit the growth of or kill bacteria which are pathogenic in nature. Citrus peels also possess diabetes lowering and antiperoxidative effect due to the high content of total polyphenols.

It is well recorded since ages in traditional Chinese medicine that the peels of the dried citrus peels are useful as remedies to ease coughs and reduce phlegm. Experiments on male rats showed that besides antiperoxidative activity, few citrus peels have antithyroidal, hypoglycemic, and insulin stimulatory properties, which suggest its potential to improve both hyperthyroidism and diabetes mellitus ${ }^{1}$.

\section{Materials and Methods}

Alloxan was purchased from the local agent of Merk (Germany) and glimepiride was a kind gift from Square Pharma. All other reagents were of analytical grade and purchased from Active Fine Chemicals Ltd.

Instruments: Glucometer (Bioland G-423 test meter, Bioland, Germany)

Preparation of coarse powder of Citrus limon peel: The fresh C. limon (lemon) was collected. The peels were separated and cut into small pieces with knife. These were dried completely under the mild sun light and ground with an electric grinder into coarse powder.

Preparation of hexane extract of C. limon: The coarse powder was submerged in hexane since this is the common solvent for extracting most of the constituents present in herbal materials. Flat bottom $500 \mathrm{ml}$ reagent bottle was used for this purpose which was kept at room temperature and allowed to stand for several days (7-10) with occasional shaking and stirring. When the solvent became concentrated, the liquid hexane was filtered 
through cotton and then through filter paper. Then solvent was allowed to evaporate at temperature $40-50^{\circ} \mathrm{C}$ and the extract of peel of $C$. limon was obtained.

Selection of animals: A total number of 20 Long Evan's female rats weighing about $150-200 \mathrm{mg}$, age 2 months were purchased from animal house of the International Center for Diarrheal Disease and Research, Bangladesh (ICDDR,B) prior to commencement of the experiment. All the rats were acclimatized to the new environment for a period of one week. During the experiment period the rats were kept in a well ventilated animal house at room temperature of $25^{\circ} \mathrm{C}$. They were supplied with standard pellets purchased from ICDDR,B and fresh drinking water. All the rats were kept in cage and maintained with natural $12 \mathrm{~h}$ light and dark cycle in the animal house of the Institute of Nutrition and Food Sciences, University of Dhaka, Bangladesh.

Grouping of experimental rats: 20 Long Evan's female rats were randomly assigned into 3 groups. Six rats in group in each group.

Group 1: The condition of diabetes was induced by using alloxan. Then these were treated with normal saline.

Group 2: The condition of diabetes was established by using alloxan. The rats of this group were treated with glimepiride.

Group 3: After inducing diabetes the rats of this group were treated with the hexane- extract of Citrus limon.

Experimental induction of diabetes: Group 1-3 animals were allowed to fast for $12 \mathrm{~h}$. Diabetes was induced by injecting intraperitoneally a freshly prepared solution of alloxan $(150 \mathrm{mg} / \mathrm{kg})$ in normal saline after base line glucose level determination. The alloxan treated animals were allowed to food over night to overcome drug induced hyperglycemia. After $48 \mathrm{hrs,} \mathrm{blood} \mathrm{glucose}$ content was measured with a Bioland G-423 test meter (Bioland, Germany) using blood sample from the tail vein of the rats. When the condition of diabetes was established in animals with blood glucose level above $11.1 \mathrm{mmol} / \mathrm{L}$ was selected for the study.

\section{Preparation of dosage of standard drug and the hexane extract of Citrus limon}

Glimepiride: Glimepiride was in powered form and freely soluble in normal saline. It was dissolved in normal saline.
Hexane extract of Citrus limon: The hexane extract of C. limon was dissolved in normal saline with small amount of ethanol by using sonicator for 10 minutes.

Administration of alloxan, glimepiride and the hexane extract of Citrus limon: The group1-3 was prepared for resting antihyperglycemic effect after alloxan induction. All the rats were starved (only water was given) for 16 hours. All the rats were tested for baseline glucose level. Group 1 was selected for diabetic control rats which did not receive extract or glimepiride. Group 2 rats were selected for glimepiride control group. Here glimepiride was administered orally at a dose of $15 \mu \mathrm{g} / \mathrm{kg}$ body weight. The rats of group 3 received orally the hexane extract of C. limon at a dose of $10 \mathrm{mg} / \mathrm{kg}$ body weight.

Collection of blood: The blood glucose level was tested by using glucometer (Bioland glucometer, Germany). In this case, the blood was collected by picking the tail vein in $0,24,48,72$ and 96 hours after the oral administration of glimepiride and lemon peel extract.

\section{Results}

In the present study diabetes was induced in rats by injecting alloxan (150 $\mathrm{mg} / \mathrm{kg}$ body weight $)$ intraperitoneally. Alloxan is a cytotoxic agent for inducing chemical diabetes (alloxan diabetes) in a wide variety of animal species by damaging insulin secreting $\beta$-cells, resulting a decrease in endogenous insulin release, which paves the way for the decreased utilization of glucose by the tissues. Hypercholesterolemia and hypertriglyceridemia are common complications of diabetes mellitus in addition to hyperglycemia.

This work has evaluated the antidiabetic effect of the hexane extract of C. limon on fasting blood glucose level in alloxan induced diabetic rats. Glimepiride has been used as standard antidiabetic agent to compare the effects of extracts for these experiments.

In case of alloxan induced diabetic rats (Figure 1), glimepiride reduced blood glucose level by $33.27 \%$, $53.37 \%, 85.06 \%$, and $92.6 \%$ in $24 \mathrm{hrs}, 48 \mathrm{hrs}, 72 \mathrm{hrs}$, and $96 \mathrm{hrs}$, respectively (Figure 2).

In case of antihyperglycemic effect of the hexane extract of C. limon, the blood glucose level was reduced to $44.57 \%, 75.96 \%, 95.43 \%$, and $98.08 \%$ in $24 \mathrm{hrs}, 48 \mathrm{hrs}$, $72 \mathrm{hrs}$, and $96 \mathrm{hrs}$, respectively (Figure 3 ).

The proposed mechanism is that, alloxan is a cytotoxic agent that causes a selective destruction of $\beta$-cell of islets of langerhans and thus produced a marked decrease of insulin secretion from the $\beta$-cells. As insulin 
lowers the blood glucose level, it is necessary to increase the secretion of insulin from the $\beta$-cells. The hexane extract of $C$. limon peel contains polyphenols which have the properties to stimulate or regenerate the $\beta$-cells for the secretion of insulin and are most effective for controlling diabetes.

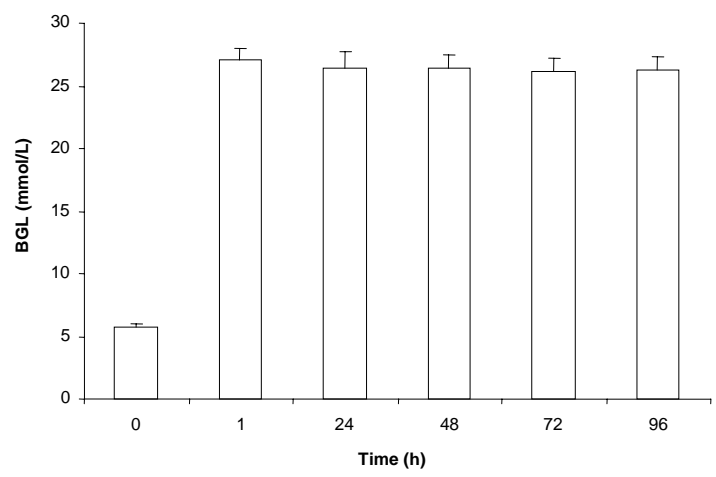

Figure 1. Blood glucose level after administration of alloxan (150 $\mathrm{mg} / \mathrm{kg}$ ). The result is expressed as mean \pm Standard Deviation, where, $\mathrm{n}=6$ in each group. BGL $=$ Blood Glucose Level

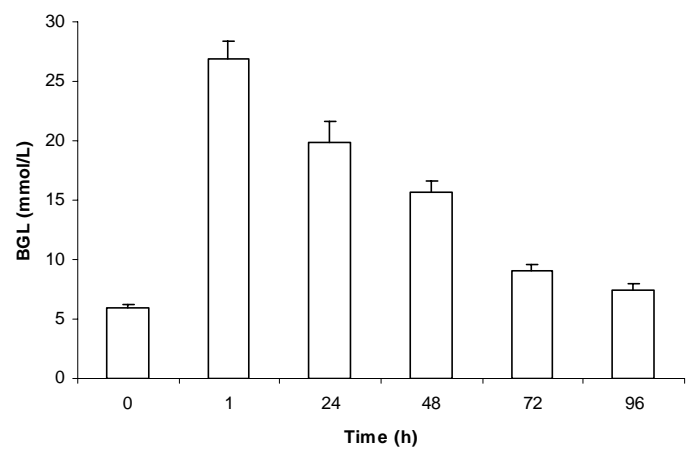

Figure 2. Blood glucose level after co-administration of alloxan (150 $\mathrm{mg} / \mathrm{kg}$ ) and glimepiride $(15 \mu \mathrm{g} / \mathrm{kg})$. The result is expressed as mean \pm Standard Deviation, where, $\mathrm{n}=6$ in each group. BGL $=$ Blood Glucose Level

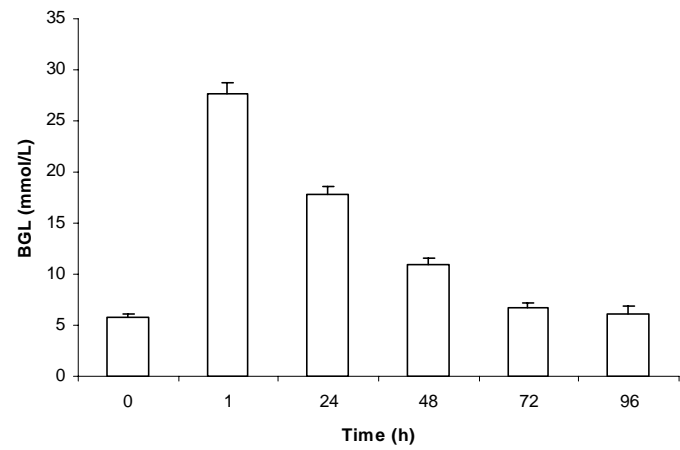

Figure 3. Blood glucose level after co-administration of alloxan (150 $\mathrm{mg} / \mathrm{kg}$ ) and hexane extract of lemon peel $(10 \mathrm{mg} / \mathrm{kg})$. The result is expressed as mean \pm Standard Deviation, where, $\mathrm{n}=6$ in each group. $\mathrm{BGL}=$ Blood Glucose Level

\section{Discussion}

Diabetes mellitus is the world's largest endocrine disease of multiple etiologies involving metabolic disorders of carbohydrate, fat and protein. At present the treatment of diabetes mainly involves a sustained reduction in hyperglycemia by the use of biguanides, thiazolidinediones, sulfonylureas, d-phenylalanine and glycosidase inhibitors in addition to insulin. However, due to unwanted side effects the efficacies of these compounds are debatable and there is a demand for new compounds for the treatment of diabetes. Hence, plants have been suggested as a rich, as yet unexplored source of potentially useful antidiabetic drugs.

The plant medicines have a long history of treating diabetes. With a disturbing rise in the prevalence of the metabolic disease and associate health care cost, interest in alternative or complementary therapies has grown. Over the last 10-20 years data from controlled investigations in animal models and patients have validated the therapeutic values of numerous phytotherapies for diabetes.

The present study has been carried out to evaluate the effects of the hexane extract of C. limon on fasting blood glucose on alloxan induced diabetic rats. Glimepiride was used as standard antidiabetic agent in this study and the following observations were obtained.

Single dose of glimepiride (Figure 2), has produced significant reduction of blood glucose level compared to normal control group in $96 \mathrm{hrs}$ of the experiment. The hexane extract of $C$. limon also significant reduction of blood glucose level in $96 \mathrm{hrs}$ of experiment exhibited (Figure 3). These results have showed that the hexane extract of C. limon significantly possess to blood glucose lowering effect in alloxan induced diabetic rats. Thus, if it is possible to isolate the active constituents from the plant extract, it will create a better opportunity for making new patented medicine for diabetic. The plant parts, therefore, seem to have promising value for the development of potent phytomedicine for diabetes. Further comprehensive phyto-pharmacological investigations are needed to isolate and characterize the exact chemical compounds responsible for hypoglycemic activity and their exact mechanism of action.

\section{Conclusion}

It can be inferred that the hexane extract of $C$. limon significantly possesses blood glucose lowering effect in 
alloxan induced diabetic rats. Thus, if it is possible to isolate the active constituents from the plant fractions, it will create new opportunity for treating diabetics from medicinal plant growing in Bangladesh.

\section{Acknowledgement}

Authors are very much grateful to Institute of Nutrition and Food Science authority for kind permission to use their animal house during the research work.

\section{References}

Salam, M.A., Baki, M.A., Azam, A.T.M.Z., Amran, M.S., Amjad, F.M., Rokeya, B. and Hossain, M.A. 2009. In vitro and in vivo effects of glipizide and gliclazide on the protein binding, plasma concentration and serum glucose, cholesterol and creatinine levels of ibuprofen, $J$. Pharmacol. Toxicol., 4, 307-313.

Mohiuddin, M., Azam, A.T.M.Z., Amran, M.S., and Hossain, M.A. 2009. In vitro study on the interaction of caffeine with gliclazide and metformin in the aqueous media, $J$. Pharmacol. Toxicol., 4, 194-204.
Islam, M.A., Akhtar, M.A., Khan, M.R.I., Hossain, M.S., Alam, A.H.M.K., Wahed, M.I.I., Amran, M.S., Rahman, B.M. and Ahmed, M. 2009. Oral glucose tolerance test (OGTT) in normal control and glucose induced hyperglycemic rats with Coccinia cordifola $L$ and catharanthus roseusL Pak. J. Pharm. Sci., 22, 402-404.

Mohiuddin, M., Amran, M.S. and Hossain, M.A. 2009. The in vivo effects of caffeine on the hypoglycemic activity of gliclazide and metformin in healthy rats. Dhaka Univ $J$. Pharm. Sci. 8, 47-51.

Mohiuddin, M., Azam, A.T.M.Z., Amran, M.S. and Hossain, M.A. In vivo effects of gliclazide and metformin on plasma concentration of caffeine in healthy rats, Pak. J. Biol. Sci., 12, 734-737.

Mohiuddin, M., Azam, A.T.M.Z., Amran, M.S. and Hossain, M.A. 2009. In vitro effects of gliclazide and metformin on protein binding of caffeine in the aqueous media, J. Biol. Sci., 9, 476-481. 